Efectos del cambio climático sobre las interacciones aveparásito

\title{
Effects of climate change on bird-parasite interactions
}

Marina García del Río ${ }^{1 *}$, Francisco Castaño-Vázquez¹, Santiago Merino¹.

(1) Departamento de Ecología Evolutiva, Museo Nacional de Ciencias Naturales-CSIC. José Gutiérrez Abascal 2. E-28006 Madrid, Spain. Telephone number: +34 914111328.

* Autor para la correspondencia: Marina García del Río, e-mail: marina049@msn.com 


\section{Resumen}

En los últimos años, los modelos sobre los efectos del cambio climático han predicho que la llegada de climas más suaves a mayores latitudes y altitudes tendrá como consecuencia la extensión de enfermedades desde latitudes y altitudes menores. Las relaciones parásito-ave no

15 son una excepción y también podrían verse afectadas por estos cambios. Sin embargo, varias revisiones del efecto del cambio climático sobre las aves y sus parásitos indican que los efectos pueden ser complejos y variados y, en general, difíciles de predecir. En este artículo repasamos algunos de los estudios que han registrado efectos climáticos sobre las relaciones ave-parásito mostrando los distintos aspectos que pueden interaccionar en estos casos. La

20 biodiversidad, la densidad de organismos, ya sean parásitos u hospedadores, la virulencia de los parásitos y la inmunocompetencia de los hospedadores, son factores clave a tener en cuenta en el impacto del cambio climático en la interacción ave-parásito. Resulta evidente que son necesarios más trabajos empíricos que muestren hasta qué punto el cambio climático puede afectar a las poblaciones de aves y parásitos y su capacidad de adaptación a dichos cambios.

Palabras clave: altitud; humedad; infección; latitud; parásitos; temperatura.

\section{Abstract}

30 During the last years models on the effects of climate change predict the arrival of milder climates to higher latitudes and altitudes, and as a consequence, the spread of disease from lower latitudes and altitudes. Bird-parasite interactions are not an exception and could be affected by these changes. However, several reviews on the effects of climate change on birds and parasites conclude that effects of climate on these interactions could be diverse and complex and, in general, difficult to predict. In this article we review some studies reporting climatic effects on bird-parasite relationships, showing different aspects that may interact in these cases. Biodiversity, density of organisms, both of hosts or parasites, virulence of parasites and host immunocompetence, are key factors to understand the effects of climate change on bird-parasite interactions. More empirical work is clearly needed to show the level of impact of climate change on birds and parasite populations as well as on their capacity of adaptation to those changes.

Keywords: altitude; humidity; infection; latitude; parasites; temperature. 
Los parásitos constituyen más de la mitad de todos los seres vivos, y desempeñan un papel ecológico particularmente importante en todos los ecosistemas. A partir de la interacción parásito-hospedador, ejercen presiones de selección extremas sobre todos los seres vivos (Price 1980). Por ello, el parasitismo es un componente importante que altera distintas fases del ciclo de vida de muchos organismos (Price 1980), incluyendo a las aves (Møller 1990, Loye y Zuk 1991, Møller 1994).

Por otro lado, el cambio climático es considerado el mayor problema ecológico de los siglos XX y XXI, con severas consecuencias en el medio ambiente (Pachauri et al. 2014). El informe realizado por el grupo intergubernamental de expertos sobre el cambio climático en 2018 (IPCC 2018), predijo que el calentamiento global probablemente superará $1.5^{\circ} \mathrm{C}$ por encima de los niveles pre-industriales para 2040 , siendo en la actualidad $1^{\circ} \mathrm{C}$ superior aproximadamente y pudiendo superar los $2^{\circ} \mathrm{C}$ en 2080 , ya que la temperatura está aumentando a razón de $0.2^{\circ} \mathrm{C}$ por década (Hoegh-Guldberg et al. 2018). Además, el aumento en la temperatura global puede ir acompañado de una mayor variabilidad en las precipitaciones con estaciones secas más severas, elevación del nivel del mar, pérdida de biodiversidad y variaciones en los rangos de distribución de los organismos (Walsh y Ryan 2000, Houghton et al. 2001, Hoegh-Guldberg et al. 2018). Uno de los referentes animales de los impactos del cambio climático son las aves, ya que su comportamiento y los cambios en sus poblaciones han sido documentados durante décadas e incluso siglos en algunos casos (Dunn y Møller 2019). Por ejemplo, se ha visto que las aves del trópico se verán más afectadas que las aves de las regiones polares (Dobson et al. 2008). Sin embargo, nuestro conocimiento sobre las respuestas ecológicas al cambio climático en diferentes organismos está aún en desarrollo debido a la incertidumbre que existe sobre los mecanismos, las trayectorias de las comunidades y ecosistemas y a que no hay evidencias de cambios en las tolerancias climáticas de una especie (Walther et al. 2002, Parmesan 2006, Teplitsky et al. 2008, Fuller et al. 2012).

Los cambios en la distribución y fenología de una variedad de taxones debidos al cambio climático, implican que los patrones estacionales de desarrollo y transmisión de muchos patógenos también se verían afectados por el clima (Harvell et al. 2002, Dobson et al. 2003, Root et al. 2003, Kutz et al. 2005). En las interacciones donde existe una estrecha dependencia de los parásitos con sus hospedadores, los efectos del cambio climático pueden afectar asimétricamente a ambos miembros de la asociación. Por lo tanto, la supervivencia de los parásitos estará determinada por varios factores, como la dependencia del hospedador y la especificidad parasitaria, la complejidad del ciclo de vida, la biodiversidad, la densidad y movilidad de sus hospedadores, y la tolerancia fisiológica de los hospedadores. Por ejemplo, las interacciones más específicas serán más susceptibles a una reducción en la población del hospedador debido a un cambio ambiental, ya que resulta más difícil la transmisión a otro hospedador que en las interacciones generalistas (Martínez y Merino 2011). Además, la disponibilidad de recursos para los hospedadores puede verse afectada por cambios 
ambientales (Chandra 1981, Merino y Møller 2010), por lo que su inmunocompetencia podría verse reducida, facilitando así la colonización y reproducción de los parásitos (Santos 1994, Christe et al. 2006, Merino 2010). Por otro lado, si los efectos del cambio climático producen desplazamientos de los hospedadores a nuevas áreas, puede darse la introducción de parásitos sin antecedentes coevolutivos previos en hospedadores nativos (Goodenough 2010). Esto podría dar lugar a reducciones drásticas en las poblaciones de estos hospedadores nativos o incluso su extinción (Warner 1968, Van Riper et al. 1986, Christe et al. 2006).

En los últimos años, varias revisiones del efecto del cambio climático sobre las aves y sus parásitos han identificado varios aspectos sobre los cuales es más probable que incidan las variables climáticas (ver por ejemplo Merino y Møller 2010, Martínez y Merino 2011, Merino 2019). Las aves, en general, son seres con gran capacidad de desplazamiento, lo que implica su presencia en un alto número de localidades $\mathrm{y}$, por tanto, una mayor exposición a diferentes parásitos (ver por ejemplo Jourdain et al. 2007), pero también la posibilidad de desplazarse a zonas con menor abundancia de parásitos. De hecho, el comportamiento migratorio de algunas aves se ha explicado no solo como una manera de evitar zonas de climas adversos y menor disponibilidad de alimentos, sino también como una manera de evitar el ataque de distintos patógenos (Altizer et al. 2011, Shaw y Binning 2016, O'Connor et al. 2018). El cambio climático ya está afectando a algunas poblaciones de aves que han limitado o alterado sus desplazamientos migratorios (ver por ejemplo Rotics et al. 2017), y esto puede también afectar a su capacidad potencial de extender patógenos a otras poblaciones. A esto se suma la capacidad de las especies invasoras de convertirse en fuente de patógenos para las poblaciones autóctonas (Warner 1968, Marzal et al. 2015) o bien de verse libres de sus patógenos específicos en las zonas donde se han introducido, disponiendo así de una ventaja frente a potenciales competidores autóctonos (Merino 2019). Alternativamente, las especies invasoras pueden verse negativamente afectadas por patógenos comunes en las zonas donde se han visto introducidas.

110 Por otro lado, los efectos del cambio climático en las interacciones parásito-hospedador son difíciles de evaluar. Por ejemplo, la disminución de las poblaciones de aves puede deberse a cambios en los factores ecológicos, como la pérdida de hábitat, y no a un efecto directo de los patógenos (Merino y Møller 2010), lo que puede causar a su vez la disminución de las poblaciones de parásitos al desaparecer sus hospedadores (Dobson et al. 2008). De hecho, el 115 cambio climático ha alterado muchos ecosistemas naturales, afectando consecuentemente a su biocenosis (Rosenzweig et al. 2007). Aunque estas interacciones tengan una gran importancia ecológica en todos los ecosistemas, pocos estudios hasta la fecha han investigado los efectos del cambio climático en la prevalencia y abundancia de los parásitos de aves, y su impacto potencial en la adecuación biológica del hospedador (Merino y Møller 2010). No 120 obstante, en estudios anteriores se ha demostrado que los factores abióticos, como la temperatura, la lluvia o la velocidad del viento, afectan a las interacciones entre ectoparásitos y 
hospedadores (Smith et al. 1998, Roulin 1999, Hubálek et al. 2003, Martínez-de la Puente et al. 2009).

Por tanto, el objetivo de este trabajo es recopilar algunos de los estudios que han registrado efectos climáticos sobre las relaciones ave-parásito, mostrando los distintos aspectos que pueden interaccionar en estos casos. Para ello, se han dividido los posibles efectos en diferentes subapartados, ya que son los más adecuados para analizar los aspectos más importantes en la relación parásito-hospedador. Esperamos poder mostrar la importancia que tienen la biodiversidad, la densidad de organismos ya sean parásitos u hospedadores, y la virulencia de los parásitos y la inmunocompetencia de los hospedadores, como factores clave a tener en cuenta en el impacto del cambio climático en la interacción ave-parásito.

\section{Efectos del clima sobre la reproducción y transmisión de los parásitos}

El aumento de las temperaturas y los cambios en el régimen de precipitaciones y en la humedad del ambiente, pueden tener efectos sobre la abundancia y prevalencia de los parásitos de aves. La densidad de parásitos, así como de vectores y hospedadores, son factores clave para la prevalencia y trasmisión de enfermedades, por lo que cualquier cambio ambiental puede alterar la interacción ave-parásito y, por lo tanto, la prevalencia de la enfermedad. En general, las precipitaciones promueven la reproducción de especies

140 dependientes de agua, como por ejemplo los mosquitos que actúan como vectores y, por otro lado, la temperatura es un factor importante para la supervivencia y desarrollo de los parásitos y sus vectores. Un aumento en las temperaturas puede acelerar la aparición de algunas etapas parasitarias, un ejemplo es el nematodo Heterakis gallinarum, parásito de Gallus gallus y especies de aves relacionadas, en el que el aumento de temperatura provoca un desarrollo

145 acelerado de las larvas y, por tanto, un aumento potencial en la prevalencia de la infección por este parásito (Saunders et al. 2000).

En estudios realizados con parásitos sanguíneos, se ha demostrado que variables climáticas relacionadas con la disponibilidad de agua no tienen efecto sobre la prevalencia, siendo las especies de hospedadores lo que determina la prevalencia de los parásitos (Pulgarín-R et al.

150 2018). Sin embargo, Sehgal et al. (2011) encontraron que en los parásitos extracelulares pertenecientes al género Trypanosoma la humedad era un indicador importante para su prevalencia, mientras que en el caso de los hemosporidios como Plasmodium la temperatura era el indicador más importante. Además, en otro estudio se comprobó que en el bisbita caminero Anthus berthelotii, las aves que habitaban en lugares con las temperaturas mínimas 155 más altas en Tenerife, tenían más probabilidades de infectarse con malaria aviar (GonzálezQuevedo et al. 2014). En general, la prevalencia de Plasmodium ha aumentado con el aumento de las temperaturas globales, especialmente en las últimas dos décadas, debido a que el aumento de temperaturas afecta a la distribución y a la incidencia de los insectos vectores (Garamszegi 2011). Además, la prevalencia de otros parásitos sanguíneos también se ha 
relacionado positivamente con la temperatura, a lo largo de un gradiente de elevación, y con la estación seca, independientemente de la altitud, en una comunidad de aves de los trópicos húmedos australianos (Zamora-Vilchis et al. 2012). Este efecto se ha explicado, tanto por el aumento de la abundancia de vectores con la temperatura, como por el desarrollo acelerado de parásitos sanguíneos dentro de los vectores a temperaturas elevadas. También se ha informado de una relación similar entre los parásitos sanguíneos y el gradiente altitudinal en Nueva Zelanda (Niebuhr et al. 2016). Por el contrario, en otro estudio realizado en la cordillera de los Andes (Colombia), se demostró que la prevalencia de otro grupo de hemosporidios, como es el género Leucocytozoon, fue mayor durante la Niña (período en el que las temperaturas de la superficie en la costa del Pacífico oriental de América del Sur son más 170 bajas que el promedio) (Mantilla et al. 2018). Esto es debido a que el género Leucocytozoon parece estar mejor adaptado para completar su ciclo de vida en climas fríos y montañosos, los cuales no son adecuados para otros géneros de parásitos (Merino et al. 1997, Merino et al. 2008, Oakgrove et al. 2014). Esto también se demostró en otro estudio en las Montañas Rocosas de Colorado, donde la prevalencia de Leucocytozoon está influenciada por los 175 cambios estacionales de temperatura al afectar a la aparición del insecto vector (simúlidos) (Murdock et al. 2013).

En estudios donde se analizaron los ectoparásitos, Meléndez et al. 2014 demostraron que la intensidad de ácaros se relacionó positivamente con la temperatura en un gradiente de elevación en cinco de seis especies de paseriformes en España. En Nuevo México, se 180 encontró una interacción positiva entre la temperatura y la sequía con la prevalencia de moscas parásitas Trypocalliphora braueri, lo cual afectaba al éxito de incubación en la especie de papamoscas cenizo (Myiarchus cinerascen) (Musgrave et al. 2019). Además, se ha demostrado la importancia de la temperatura del nido de las aves como una señal utilizada por los insectos para localizar los nidos de sus hospedadores (Martínez-de la Puente et al. 2010).

185 En nidos de golondrinas, Dawson et al. (2005) encontraron una relación curvilínea en la abundancia de larvas parásitas (hematófagas) de pollos de Protocalliphora con la temperatura y, por otro lado, Bennett y Whitworth (1991) encontraron una aceleración en la pupación, pero no en la metamorfosis, debido al aumento de la temperatura, y que su supervivencia disminuyó conforme aumentó la humedad. Sin embargo, aunque los modelos de cambio climático 190 predicen que las enfermedades se propagarán con el aumento de las temperaturas (Patz et al. 1996, Lafferty y Mordecai 2016, Wu et al. 2016), estudios recientes concluyeron que un aumento en la temperatura hizo disminuir la abundancia de ectoparásitos que habitan en los nidos de aves (Mennerat et al. 2019). En concreto, Castaño-Vázquez y colaboradores (2018) demostraron que la abundancia de Protocalliphora azurea y el ácaro Dermanyssus gallinoides 195 se redujeron significativamente a consecuencia de un aumento en la temperatura, mientras que otras poblaciones de parásitos no se vieron afectadas, y concluyeron que el efecto de la temperatura sobre la humedad podría haber mediado estos efectos, dado que algunos artrópodos son muy sensibles a la pérdida de agua. Esto concuerda con los resultados de Garrido-Bautista et al. 2020, quienes demostraron que en ambientes mediterráneos la mayor 
prevalencia de Protocalliphora azurea se da en hábitats húmedos. Esto se puede explicar ya que en los parásitos, sobre todo aquellos con etapas de vida libre, los factores abióticos pueden ser especialmente importantes para su desarrollo y transmisión (Mouritsen y Poulin 2002, Ogden et al. 2006, Poulin y Mouritsen 2005). Por tanto, las especies de Protocalliphora y el sistema ave-parásito se pueden ver afectados de diferente forma según el hábitat geográfico,

205 ya que estos parásitos probablemente estén adaptados localmente. Sin embargo, otros estudios concluyeron que el efecto aparente de la temperatura en el aumento en la abundancia de los parásitos fue mediado por un efecto en la reproducción y condición corporal de los hospedadores (Møller et al. 2013). También Dudaniec y colaboradores (2007) concluyeron que los cambios en la prevalencia del díptero Philornis downsi no se debían a un efecto directo del 210 clima sobre el parásito. Sin embargo, otros estudios de $P$. downsi demostraron que su prevalencia era mayor en regiones húmedas de las islas Galápagos (Wiedenfeld et al. 2007), siendo al contrario en la isla Santa Cruz (Dudaniec et al. 2007), y Koop et al. (2013) concluyó que dicha especie de parásito era capaz de resistir las fluctuaciones climáticas extremas características del hábitat de estas Islas, contrastando así estudios anteriores donde se vio que

215 las sequías le afectan y usan los hábitats húmedos como reservorios (Fessl et al. 2006A, Fessl et al. 2006B). Por otro lado, se ha demostrado que una primavera lluviosa y fría afecta negativamente la abundancia de ciertos ectoparásitos de aves (Merino y Potti 1996), y que una temperatura máxima promedio alta y un aumento en las precipitaciones se correlacionaron positivamente con la intensidad media de Philornis torquans en aves argentinas (Antoniazzi et 220 al. 2011). Del mismo modo, se ha observado que cambios en la humedad en el interior de las cajas nido pueden regular la estructura poblacional y sucesión de ciertas especies de ectoparásitos de aves (Heeb et al. 2000). Los ambientes húmedos pueden favorecer un aumento de la carga de ectoparásitos en las aves, como ocurre en la paloma bravía (Columba livia) (Moyer et al. 2002). El régimen de lluvias puede alterar la densidad de vectores, ya que un 225 aumento en las precipitaciones puede promover la reproducción de especies dependientes del agua. Esto sucede en diferentes especies de mosquitos cuya abundancia está asociada con la prevalencia de las enfermedades que transmiten (Gill 1938, Wegbreit y Reisen 2000, Tong y Hu 2001, Zhou et al. 2004, Shaman y Day 2007). Por último, se ha observado que otros factores abióticos como la velocidad del viento también pueden afectar negativamente a la 230 abundancia de algunos ectoparásitos, como ocurre con los simúlidos (Martínez-De La Puente et al. 2009). Las diferentes relaciones entre las variables climáticas y los parásitos y sus vectores mencionados en este apartado están recogidos en la tabla 1.

\section{Efectos del clima sobre los ajustes de los ciclos vitales de los parásitos}

235 Los inviernos más cálidos o las primaveras tempranas debidos al cambio climático en determinadas regiones geográficas, pueden alterar la fenología de los parásitos y sus hospedadores. Por ejemplo, la reproducción de los hospedadores puede ajustarse por la 
temperatura y las precipitaciones, que a su vez determinan la disponibilidad de alimento. Además, los cambios en el clima pueden alterar la magnitud y el momento de la transmisión 240 máxima de los parásitos, lo que afecta también a los hospedadores (Polley y Thompson 2009). Un ejemplo de ello se da en partes del norte de Europa, donde el avance de la primavera debido a un aumento de la temperatura ha afectado, además de a la abundancia, a la fenología de los parásitos. Por ejemplo, el avance en la fenología de la mosca Ornithomyia avicularia, se asoció con una mayor prevalencia de este parásito en las golondrinas Hirundo rustica (Møller 245 2010). Otro ejemplo es la influencia de la temperatura durante los diferentes períodos estacionales en la aparición y en el fin de la diapausa de la mosca hematófaga Carnus hemapterus, parásita de aves que nidifican en cavidades (Calero-Torralbo 2011, Amat-Valero et al. 2013).

Por otro lado, se ha demostrado que, junto con pequeños mamíferos y humanos, las aves 250 actúan como reservorios de las garrapatas (Ixodes scapularis), principal vector del agente que causa la enfermedad de Lyme (Borrelia burgdorferi) en el noreste de América del Norte, contribuyendo así a su propagación (Newman et al. 2015). Levi y colaboradores (2015) mostraron que en pequeños mamíferos la fenología de las ninfas y larvas de las garrapatas avanzó durante los años en que mayo y agosto fueron más cálidos, sin embargo no hubo una 255 mayor sincronía entre ambas fases de vida, es decir, el tiempo en el que las ninfas y las larvas se alimentan simultáneamente. Por lo que, debido a que la sincronía entre las ninfas y las larvas favorece la transmisión de patógenos que producen infecciones de corta duración, el cambio climático puede aumentar o mantener la transmisión de patógenos persistentes, pero puede inhibir los de corta duración. Además, otro estudio con dicha especie de garrapatas en 260 ratones, concluyó que los patógenos persistentes fueron más aptos para las predicciones de cambio climático de 2020, 2050 y 2080. Sin embargo, teniendo en cuenta los patrones de actividad estacional de las garrapatas estimados para 2020 y 2050, los patógenos más aptos fueron los menos persistentes, por lo que el cambio climático a través de los efectos de la temperatura sobre la estacionalidad de la garrapata I. scapularis puede afectar los procesos evolutivos de los patógenos que transmite (Ogden et al. 2008).

El avance en las fechas de puesta y los cambios en los parámetros de reproducción de las aves por el cambio climático reciente, ha afectado a las relaciones ave-parásito de cría, por ejemplo, se ha visto que los hospedadores migrantes de corta distancia del cuco Cuculus canorus llegaron antes a las zonas de reproducción debido al aumento de las temperaturas,

270 por lo que su nivel de parasitismo disminuyó (Saino et al. 2009). Y, por el contrario, se ha visto que en aves migrantes de larga distancia que adelantaron su reproducción presentaron más casos de parasitismo por Cuculus canorus (Dyrcz y Czyz 2018). Estos datos respaldan la hipótesis de que el cambio de hospedador por parte del cuco común está impulsado, al menos parcialmente, por el cambio climático (Møller et al. 2011). Además, en otros estudios se ha 275 visto que el cambio climático afecta al tamaño de los huevos de los cucos y de sus hospedadores (Berkowic et al. 2015), y que la variación temporal y geográfica en los factores 
ambientales puede afectar al mimetismo de los huevos del cuco (Møller y Soler 2012). Sin embargo, los desajustes fenológicos observados en el críalo europeo (Clamator glandarius) no parecen deberse a la respuesta de sus hospedadores a las condiciones climáticas (Avilés et al. 2014). Tampoco es el caso del cuco común en Noruega, donde se mostró su llegada anticipada a las zonas de reproducción pero no la de sus hospedadores, por lo que no se encontró evidencia de que cambios en el clima provocasen un desajuste fenológico entre el parásito y sus hospedadores que contribuyera a la disminución de su población (Barrett 2014).Los efectos en las fenologías de los diferentes organismos mencionados debidos al cambio climático se resumen en la tabla 2.

\section{¿Contribuye el clima a la extensión de las enfermedades en o por las aves?}

El aumento de la temperatura y los cambios en la humedad en ciertas áreas geográficas no solo puede afectar a la abundancia y la fenología de los parásitos y sus hospedadores, sino también a sus áreas de distribución, ya que dichas áreas pueden volverse climáticamente favorables para ciertas especies debido al cambio climático. El caso de las aves es especialmente relevante, ya que son altamente móviles y pueden expandir su rango de distribución más fácilmente en comparación con otros vertebrados, exceptuando aquellas especies filopátricas con escasa dispersión desde su área natal. El rango de distribución de los vectores también está relacionado con la temperatura y las precipitaciones, por lo que sus rangos de distribución pueden aumentar si cambian las condiciones climáticas. Un ejemplo es la expansión de la garrapata $I$. scapularis en Canadá por parte de, presumiblemente, aves migratorias y hospedadores residentes, que ha extendido la enfermedad de Lyme en dicha región (Leighton et al. 2012). Un caso similar ocurrió en Canadá con la toxoplasmosis, enfermedad que se propagó debido a una expansión por parte de felinos silvestres, los cuales aumentaron la diseminación de oocistos de Toxoplasma gondii en el ambiente (Jenkins et al. 2013). Esto, unido a una temperatura cada vez más alta, pudo aumentar el potencial de infección de hospedadores intermedios, como aves y varios mamíferos (Reiling y Dixon 2019). Otro estudio concluyó que las aves migratorias tienen la capacidad de aumentar el alcance de las garrapatas incluso a regiones que actualmente no son adecuadas climáticamente para su establecimiento. Por lo tanto, teniendo en cuenta el aumento de temperatura según los escenarios de cambio climático, se espera que las garrapatas puedan extender su alcance a más regiones del norte de Canadá en el futuro (Ogden et al. 2015). Además, en Europa, se ha demostrado que las garrapatas Ixodes ricinus infectadas por diversas especies de Borrelia son transportadas por paseriformes migrantes a latitudes más altas (Hasle et al. 2011).

Por otro lado, la expansión del rango de distribución de ciertos vectores como los nematóceros, también se ha relacionado con el aumento de temperaturas. Por ejemplo, se ha producido una expansión a altitudes mayores en las islas de Hawái y en Australia (Atkinson 2008, Lovejoy 2008, Zamora-Vilchis et al. 2012, Atkinson et al. 2014, Liao et al. 2015) y a latitudes mayores 
en Nueva Zelanda (Tompkins y Gleeson 2006). En ambos casos, el cambio de distribución de los mosquitos vectores (Culex quinquefasciatus) estaría asociado con un aumento en la prevalencia de enfermedades que transmiten en concreto la malaria aviar (Plasmodium sp.). En general, debido al aumento de las temperaturas se esperan cambios latitudinales y altitudinales en el rango de la malaria aviar. Por ejemplo, en distintas regiones geográficas como son Alaska y Francia, ha habido y se espera un aumento en latitud de la malaria aviar (Loiseau et al. 2012, Loiseau et al. 2013). Cabe destacar que un estudio realizado por LaPointe y colaboradores (2010) demostró que las bajas temperaturas fueron las responsables, y no la distribución del vector, de prevenir el desarrollo de los esporozoitos y, por tanto, la transmisión de la malaria aviar. Esto concuerda con el estudio de Merino y colaboradores (2008), en el que se encontró 325 una relación negativa entre prevalencia y latitud en linajes de Haemoproteus, Plasmodium e infecciones mixtas. Sin embargo, esta relación fue positiva en el caso de Leucocytozoon. Esta relación entre los parásitos del género Leucocytozoon y latitudes altas, ya fue demostrada previamente en otras regiones (Greiner et al. 1975). La prevalencia de este género de parásitos también aumenta con la altitud (van Rooyen et al. 2013, González et al. 2014), por lo que un aumento de temperaturas en zonas de latitudes y/o altitudes mayores pueden disminuir su rango de distribución. En el caso de otros parásitos sanguíneos extracelulares, como las microfilarias o los pertenecientes al género Trypanosoma, la prevalencia disminuye según aumenta la altitud (Chapa-Vargas et al. 2020).

Debido al aumento de temperaturas en zonas con altitudes y/o latitudes altas, la distribución de los vectores puede cambiar hacia esas zonas y, por tanto, la de los parásitos que son trasmitidos por ellos (Zamora-Vilchis et al. 2012). Esto implica que las zonas con latitudes/ altitudes mayores pueden llegar a ser más susceptibles a los efectos del cambio climático sobre los parásitos. Los diferentes efectos del cambio climático sobre los rangos de distribución de los organismos mencionados se recogen en la tabla 3. Por otro lado, hay que tener en cuenta también la existencia de barreras geográficas, las cuales pueden aislar poblaciones de vectores, aves o parásitos, dificultando la propagación de enfermedades a latitudes mayores. Algunos ejemplos de barreras geográficas son los desiertos alrededor de áreas tropicales (Barrientos et al. 2014, Martínez et al. 2016, Ayadi et al. 2018), las grandes cadenas montañosas, las islas y la diferente proporción de superficie oceánica y terrestre entre los dos 345 hemisferios (Chown et al. 2004). Además, las variaciones en la distribución geográfica de los parásitos sanguíneos son altamente dependientes de las características de los vectores y de sus adaptaciones a los factores abióticos del ecosistema, lo cual también afecta a la velocidad de desarrollo de los parásitos dentro del vector (LaPointe et al. 2010, Morand 2015). De hecho, se ha podido constatar que la mortalidad de las aves en zonas tropicales debido a los efectos de los parásitos puede llegar a ser un $20 \%$ superior que aquellas de zonas templadas (Møller y Garamszegi 2010). 


\section{El clima puede afectar a la virulencia de los parásitos y a la inmunocompetencia de las}

aves

Debido al aumento de la abundancia y expansión del rango de distribución de los parásitos por el cambio climático, la virulencia de estos, es decir, el efecto neto de los parásitos en los hospedadores, puede verse afectada. Esto es porque la virulencia puede aumentar en parásitos que infectan a hospedadores recién llegados a áreas nuevas, o, por el contrario, se pueden introducir nuevos parásitos que tengan una virulencia mayor en los hospedadores nativos de estas áreas, ya que no se han enfrentado previamente a los parásitos recién llegados (Merino 2013). En ambos casos, el aumento de la virulencia puede darse debido a que las temperaturas más cálidas permiten que los parásitos completen sus ciclos de vida más rápidamente, aumentando así su densidad (Marcogliese 2001, Santiago-Alarcón et al. 2012, Zamora-Vilchis et al. 2012). Otra posibilidad es que el clima afecte negativamente a las aves hospedadoras reduciendo, por ejemplo, la disponibilidad de alimento y provocando un aumento del estrés y consumo de energía, que pueden conducir a la inmunosupresión y mayor susceptibilidad a diversas enfermedades (Merino y Møller 2010).

Møller y colaboradores (2013) sugieren que los parásitos, debido a un efecto indirecto de la temperatura, afectan a la fecundidad y condición corporal de sus hospedadores. También en el caso del virus del Nilo Occidental se ha visto que nuevas cepas altamente virulentas han evolucionado después de la expansión de su rango de distribución (Kilpatrick 2011). Sin embargo, en otros estudios se ha observado que un aumento de la temperatura ha reducido las poblaciones de parásitos o su actividad, produciendo así una menor virulencia de los mismos. Un ejemplo de ello son las garrapatas, que a temperaturas elevadas reducen su actividad de búsqueda de hospedadores (Randolph 2009, Dantas-Torres y Otranto 2013). Por otro lado, también se ha observado que cambios en la estacionalidad de las precipitaciones pueden afectar la especificidad de varios parásitos sanguíneos (Fecchio et al. 2019) y que, debido al cambio climático, podemos esperar la extinción de los parásitos especialistas y la expansión de los generalistas (Carlson et al. 2017), pudiendo llegar a infectar mayor número de especies y así aumentar su virulencia.

Con respecto a la inmunocompetencia de las aves, se ha visto que tanto el aumento como la disminución de la temperatura corporal afecta a su respuesta inmune. Aunque la aclimatación previa a las condiciones térmicas elimina el efecto inmunosupresor del estrés (Martínez y Merino 2011), varios estudios han relacionado la aparición de brotes epidémicos con

385 fluctuaciones extremas de temperatura (Travers et al. 2008, Wegner et al.2008, McClanahan et al. 2009). En aves, se ha demostrado que la relación entre su inmunidad y la temperatura es indirecta y está mediada por la abundancia del parásito (Zamora-Vilchis et al. 2013).

\section{Conclusiones}


Los efectos del cambio climático sobre las interacciones parásito-ave son diversos y difíciles de pronosticar. Esto es debido a que dichos efectos dependen de los factores bióticos y abióticos del hábitat donde se desarrolla la interacción, las características ecológicas y fisiológicas del parásito y su hospedador, y de las características específicas de la propia interacción (Martínez y Merino 2011). Por lo tanto, para poder evaluar los efectos del cambio climático sobre las interacciones parásito-ave se debe tener un conocimiento amplio sobre los factores que puedan modular dichas interacciones.

Como resultado de esta revisión se puede afirmar que los cambios ambientales producidos por el cambio climático generan una alteración en los ecosistemas a través de tres factores fundamentales: la biodiversidad, la densidad de los parásitos y los hospedadores, y la virulencia de los parásitos y la inmunocompetencia de los hospedadores. Por consiguiente, la tolerancia a los cambios ambientales será la que determine el grado de impacto de estos tres factores mencionados, lo cual determinará el efecto sobre la interacción parasitaria. Por último, para comprender cómo las aves se adaptarán a la presencia de nuevos parásitos en sus áreas de distribución, se hacen necesarios estudios con especies parásitas invasoras, así como estudios que analicen el efecto de la degradación de los ecosistemas, la perdida de hábitat y la contaminación, como consecuencias del cambio climático y de las actividades antropogénicas, y, además, realizar estudios experimentales que ayuden a determinar los efectos ambientales locales en las interacciones parásito-ave. 
Altizer, S., Bartel, R., Han, B. A. 2011. Animal migration and infectious disease risk. Science 331(6015):296-302.

Amat-Valero, M., Calero-Torralbo, M.A., Valera, F. 2013. Temperature during the free-living phase of an ectoparasite influences the emergence pattern of the infective phase. Parasitology 140(11):1357-1367.

Antoniazzi, L.R., Manzoli, D.E., Rohrmann, D., Saravia, M.J., Silvestri, L., Beldomenico, P.M. 2011. Climate variability affects the impact of parasitic flies on Argentinean forest birds. Journal of Zoology 283(2):126-34.

Atkinson, C.T. 2008. Avian malaria. En: Atkinson, C.T., Thomas, N.J., Hunter, B. (eds.), 420 Parasitic Diseases of Wild Birds, pp. 35-53. Wiley-Blackwell, lowa, Estados Unidos de América.

Atkinson, C.T., Utzurrum, R.B., LaPointe, D.A., Camp, R.J., Crampton, L.H., Foster, J.T., Giambelluca, T.W. 2014. Changing climate and the altitudinal range of avian malaria in the Hawaiian Islands: an ongoing conservation crisis on the island of Kaua'i. Global Change Biology 20(8):2426-2436.

Avilés, J.M., Molina-Morales, M., Martínez, J.G. 2014. Climatic effects and phenological mismatch in cuckoo host interactions: a role for host phenotypic plasticity in laying date? Oikos 123(8):993-1002.

Ayadi, T., Selmi, S., Hammouda, A., Reis, S., Boulinier, T., Loiseau, C. 2018. Diversity, prevalence and host specificity of avian parasites in southern Tunisian oases. Parasitology 145(7):971-978.

Barrett, R.T. 2014. Has climate change resulted in a mismatch between the spring arrival of the common cuckoo Cuculus canorus and its hosts in North Norway? Ornis Norvegica 37: $11-14$.

Barrientos, R., Valera, F., Barbosa, A., Carrillo, C. M., Moreno, E. 2014. Biogeography of haemo-and ectoparasites of an arid-land bird, the Trumpeter finch. Journal of arid environments 106:11-17.

Bennett, G.F., Whitworth, T.L. 1991. Studies on the life history of some species of Protocalliphora (Diptera: Calliphoridae). Canadian Journal of Zoology 69(8):2048-2058.

440 Berkowic, D., Stokke, B.G., Meiri, S., Markman, S. 2015. Climate change and coevolution in the cuckoo-reed warbler system. Evolutionary Ecology 29(4):581-597. 
Calero-Torralbo, M. 2011. Factores ecológicos y mecanismos implicados en la variabilidad de la interacción entre un ectoparásito generalista (Carnus hemapterus) y sus hospedadores. Tesis de Doctorado, Universidad de Granada, España.

Carlson, C.J., Burgio, K.R., Dougherty, E.R., Phillips, A.J., Bueno, V.M., Clements, C.F., et al. 2017. Parasite biodiversity faces extinction and redistribution in a changing climate. Science advances 3(9):e1602422.

Castaño-Vázquez, F., Martínez, J., Merino, S., Lozano, M. 2018. Experimental manipulation of temperature reduce ectoparasites in nests of blue tits (Cyanistes caeruleus). Journal of Avian Biology 49(8):e01695.

Chandra, R.K. 1981. Immunocompetence as a functional index of nutritional status. British Medical Bulletin 37(1):89-94.

Chapa-Vargas, L., Matta, N.E., Merino, S. 2020. Effects of ecological gradients on tropical avian haemoparasites. Chapter 8. En: Santiago-Alarcón, D., Marzal Reynolds, A. (eds.) Avian malaria and related parasites in the Neotropical region: ecology, evolution, and systematics. Springer: en prensa.

Chown, S.L., Sinclair, B.J., Leinaas, H.P., Gaston, K.J. 2004. Hemispheric asymmetries in biodiversity: a serious matter for ecology. PLoS biology 2(11).

Christe, P., Morand, S., Michaux, J. 2006. Biological conservation and parasitism. En: Morand, S., Krasnov, B.R., Poulin, R. (eds.), Micromammals and Macroparasites: From Evolutionary Ecology to Management, pp. 593-613. Dusseldorf, Springer, Alemania.

Dantas-Torres, F., Otranto, D. 2013. Seasonal dynamics of Ixodes ricinus on ground level and higher vegetation in a preserved wooded area in southern Europe. Veterinary parasitology 192(1-3):253-258.

Dawson, R.D, Hillen, K.K., Whitworth, T.L. 2005. Effects of experimental variation in temperature on larval densities of parasitic Protocalliphora (Diptera: Calliphoridae) in nests of tree swallows (Passeriformes: Hirundinidae). Environmental Entomology 34(3):563-568.

Dobson, A., Kutz, S., Pascual, M., Winfree, R. 2003. Pathogens and parasites in a changing climate. En: Hannah, L. and Lovejoy, T. (eds.), Climate change and biodiversity: synergistic impacts, pp. 33-38. Washington DC, Estados Unidos de América.

Dobson, A., Lafferty, K.D., Kuris, A.M., Hechinger, R.F., Jetz, W. 2008. Homage to Linnaeus: how many parasites? How many hosts? PNAS 105:11482-11489. 
Dudaniec, R., Fessl, B., Kleindorfer, S. 2007. Interannual and interspecific variation in intensity 475 of the parasitic fly, Philornis downsi, in Darwin's finches. Biological Conservation 139(34):325-332.

Dunn, P.O., Møller, A.P. (eds.). 2019. Effects of Climate Change on Birds. Oxford University Press, Oxford, Reino Unido.

Dyrcz, A., Czyz, B. 2018. Advanced breeding time in line with climate did not affect productivity 480 of great reed warblers Acrocephalus arundinaceus despite the shortening of the nestling period. Acta Ornithologica 53(1):13-22.

Fecchio, A., Bell, J.A., Pinheiro, R.B., Cueto, V.R., Gorosito, C.A., Lutz, H.L., et al. 2019. Avian host composition, local speciation and dispersal drive the regional assembly of avian malaria parasites in South American birds. Molecular ecology 28(10):2681-2693.

Fessl, B., Kleindorfer, S., Tebbich, S. 2006A. An experimental study on the effects of an introduced parasite in Darwin's finches. Biological Conservation 127(1):55-61.

Fessl, B., Sinclair, B.J., Kleindorfer, S. 2006B. The life-cycle of Philornis downsi (Diptera: Muscidae) parasitizing Darwin's finches and its impacts on nestling survival. Parasitology 133(6):739-747.

490 Fuller, T., Bensch, S., Müller, I., Novembre, J., Pérez-Tris, J., Ricklefs, R.E., et al. 2012. The ecology of emerging infectious diseases in migratory birds: an assessment of the role of climate change and priorities for future research. Ecohealth 9(1):80-8.

Garamszegi, L.Z. 2011. Climate change increases the risk of malaria in birds. Global Change Biology 17(5):1751-9.

495 Garrido-Bautista, J., Moreno-Rueda, G., Baz, A., Canal, D., Camacho, C., Cifrián, B., et al. 2020. Variation in parasitoidism of Protocalliphora azurea (Diptera: Calliphoridae) by Nasonia vitripennis (Hymenoptera: Pteromalidae) in Spain. Parasitology Research 119(2):559-566.

Gill, C.A. 1938. The Seasonal Periodicity of Malaria and the Mechanism of the Epidemic Wave. J\&A Churchill, Londres, Reino Unido.

González-Quevedo, C., Davies, R.G., Richardson, D.S. 2014. Predictors of malaria infection in a wild bird population: landscape-level analyses reveal climatic and anthropogenic factors. Journal of Animal Ecology 83(5):1091-102.

González, A.D., Matta, N.E., Ellis, V.A, Miller, E.T., Ricklefs, R.E., Gutierrez, H.R. 2014. Mixed species flock, nest height, and elevation partially explain avian haemoparasite prevalence in Colombia. PLoS One 9(6):e100695. 
Goodenough, A.E. 2010. Are the ecological impacts of alien species misrepresented? A review of the "native good, alien bad" philosophy. Community Ecology 11(1):13-21.

Greiner, E.C., Bennett, G.F., White, E.M., Coombs, R.F. 1975. Distribution of the avian hematozoa of North America. Canadian Journal of Zoology 53(12):1762-1787.

Harvell, C.D., Mitchell, C.E., Ward, J.R., Altizer, S., Dobson, A.P., Ostfeld, R.S., Samuel, M.D. 2002. Climate warming and disease risks for terrestrial and marine biota. Science 296(5576):2158-2162.

Hasle, G., Bjune, G.A., Midthjell, L., Røed, K.H., Leinaas, H.P. 2011. Transport of Ixodes ricinus infected with Borrelia species to Norway by northward-migrating passerine birds. Ticks and Tick Borne Diseases 2(1):37-43.

Heeb, P., Köliker, M., Richner, H. 2000. Bird-Ectoparasite interactions, nest humidity, and ectoparasite community structure. Ecology 81(4):958-968.

Hoegh-Guldberg, O., Jacob, D., Taylor, M., Bindi, M., Brown, S., Camilloni, I., et al. 2018. Impacts of $1.5 \mathrm{C}$ global warming on natural and human systems. En: Intergovernmental Panel on Climate Change (IPCC) Special Report on the Impacts of Global Warming of $1.5^{\circ} \mathrm{C}$ above Pre-industrial Levels and Related Global Greenhouse Gas Emission Pathways, in the Context of Strengthening the Global Response to the Threat of Climate Change, Sustainable Development, and Efforts to Eradicate Poverty. World Meteorological Organization, Geneva, Suiza.

Houghton, J.T., Ding, Y., Grigggs, D.J., Noguer, M., van der Linden, P.J., Dai, X., et al. 2001. IPCC third assessment report: climate change 2001. Cambridge University Press, Cambridge, Reino Unido.

Hubálek, Z., Halouzka, J., Juøicová, Z. 2003. Host-seeking activity of ixodid ticks in relation to weather variables. Journal of Vector ecology 28(2):159-165.

Jenkins, E.J., Castrodale, L.J., de Rosemond, S.J., Dixon, B.R., Elmore, S.A., Gesy, K.M., et al. 2013. Tradition and transition: parasitic zoonoses of people and animals in Alaska, northern Canada, and Greenland. Advances in parasitology 82:33-204.

Jourdain, E., Gauthier-Clerc, M., Bicout, D., Sabatier, P. 2007. Bird Migration Routes and Risk for Pathogen Dispersion into Western Mediterranean Wetlands. Emerging Infectious Diseases 13(3):365-372.

Kilpatrick, A.M. 2011. Globalization, land use, and the invasion of West Nile Virus. Science 334(6054):323-7.

Koop, J.A.H., Le Bohec, C., Clayton, D.H. 2013. Dry year does not reduce invasive parasitic fly prevalence or abundance in Darwin's finch nests. Reports in Parasitology 3:11-17. 
Kutz, S.J., Hoberg, E.P., Polley, L., Jenkins, E.J. 2005. Global warming is changing the dynamics of Arctic host-parasite systems. Proceedings of the Royal Society B: Biological Sciences 272(1581):2571-2576.

Lafferty, K.D., Mordecai, E.A. 2016. The rise and fall of infectious disease in a warmer world. F1000Research 5.

LaPointe, D.A., Goff, M.L., Atkinson, C.T. 2010. Thermal constraints to the sporogonic development and altitudinal distribution of avian malaria Plasmodium relictum in Hawai'i. Journal of Parasitology 96(2):318-324.

Leighton, P.A., Koffi, J.K., Pelcat, Y., Lindsay, L.R., Ogden, N.H. 2012. Predicting the speed of tick invasion: an empirical model of range expansion for the Lyme disease vector Ixodes scapularis in Canada. Journal of Applied Ecology 49(2):457-64.

Levi, T., Keesing, F., Oggenfuss, K., Ostfeld, R.S. 2015. Accelerated phenology of blacklegged ticks under climate warming. Philosophical Transactions of the Royal Society of London Series B: Biological Sciences 370(1665):20130556.

555 Liao, W., Timm, O.E, Zhang, C., Atkinson, C.T., LaPointe, D.A., Samuel, M.D. 2015. Will a warmer and wetter future cause extinction of native Hawaiian Forest birds? Global Change Biology 21(12):4342-52.

Loiseau, C., Harrigan, R.J., Bichet, C., Julliard, R., Garnier, S., Lendvai, A.Z., Chastel, O., Sorci, G. 2013. Predictions of avian Plasmodium expansion under climate change. Scientific Reports 3(1):1126.

Loiseau, C., Harrigan, R.J., Cornel, A.J., Guers, S.L., Dodge, M., Marzec, T., et al. 2012. First evidence and predictions of Plasmodium transmission in Alaskan bird populations. PLoS One 7(9):e44729.

Lovejoy, T. 2008. Climate change and biodiversity. Revue scientifique et technique (International Office of Epizootics) 27(2):331-338.

Loye, J.E., Zuk, M. 1991. Bird-parasite interactions: ecology, evolution and behaviour. Oxford University Press, Oxford, Reino Unido.

Mantilla, J.S., Moncada, L.I., Matta, N.E., Adler, P.H. 2018. Distribution of black flies (Diptera: Simuliidae) along an elevational gradient in the Andes Mountains of Colombia during the El Niño Southern Oscillation. Acta tropica 183:162-172.

Marcogliese, D.J. 2001. Implications of climate change for parasitism of animals in the aquatic environment. Canadian Journal of Zoology 79(8):1331-52. 
Martínez-de la Puente, J., Merino, S., Lobato, E., Rivero de Aguilar, J., del Cerro, S., Ruiz de Castañeda, R., Moreno, J. 2009. Does weather affect biting fly abundance in avian nests?. Journal of Avian Biology 40(6):979-987.

Martínez-de la Puente, J., Merino, S., Lobato, E., Rivero-de Aguilar, J., del Cerro, S., Ruiz de Castañeda, R., Moreno, J. 2010. Nest-climatic factors affect the abundance of biting flies and their effects on nestling condition. Acta Oecologica 36(6):543-547.

Martínez, J., Merino, S. 2011. Host-parasite interactions under extreme climatic conditions. Current Zoology 57(3):390-405.

Martínez, J., Vásquez, R.A., Marqués, A., Díez-Fernández, A., Merino, S. 2016. The prevalence and molecular characterisation of blood parasites infecting the vulnerable tamarugo conebill (Conirostrum tamarugense) and other birds in the Pampa del Tamarugal, Chile. Emu-Austral Ornithology 116(3):310-314.

Marzal, A., García-Longoria, L., Callirgos, J.M.C., Sehgal, R.N. 2015. Invasive avian malaria as an emerging parasitic disease in native birds of Peru. Biological Invasions 17(1):39-45.

McClanahan, T.R., Weil, E., Maina, J. 2009. Strong relationship between coral bleaching and growth anomalies in massive Porites. Global Change Biology 15(7):1804-1816.

Meléndez, L., Laiolo, P., Mironov, S., García, M., Magaña, O., Jovani, R. 2014. Climate-driven 590 variation in the intensity of a host-symbiont animal interaction along a broad elevation gradient. PLoS One 9:e101942.

Mennerat, A., Charmantier, A., Hurtrez-Bousses, S., Perret, P., Lambrechts, M.M. 2019. Parasite intensity is driven by temperature in a wild bird. bioRxiv: 323311.

Merino, S. 2010. Immunocompetence and parasitism in nestlings from wild populations. The 595 Open Ornithology Journal 3(1):27-32.

Merino, S. 2013. Diseñados por la enfermedad. El papel del parasitismo en la evolución de los seres vivos. Síntesis, Madrid, España.

Merino, S. 2019. Host-parasite interactions and climate change. Chapter 14. En: Dunn, P.O, Møller, A.P. (eds.) Effects of Climate Change on Birds. 2nd Edition, pp. 187-198. Oxford University Press, Nueva York, Estados Unidos de América.

Merino, S., Møller, A.P. 2010. Host-parasite interactions and climate change. En: Møller, A.P., Fiedler, W., Berthold, P. (eds.), Effects of Climate Change on Birds, pp. 213-226. Oxford University Press, Nueva York, Estados Unidos de América. 
Merino, S., Moreno, J., Vásquez, R.A., Martínez, J., Sánchez-Monsálvez, I., Estades, C.F. et al. 605 2008. Haematozoa in forest birds from southern Chile: latitudinal gradients in prevalence and parasite lineage richness. Austral Ecology 33(3):329-340.

Merino, S., Potti, J. 1996. Weather dependent effects of ectoparasites on their bird host. Ecography 19(2):107-113.

Merino, S., Potti, J., Fargallo, J.A. 1997. Blood parasites of passerine birds from central Spain. Journal of Wildlife Diseases 33(3):638-641.

Møller. A.P. 1990. Effects of parasitism by the haematophagous mite Ornithonyssus bursa on reproduction in the barn swallow (Hirundo rustica). Ecology 71(6):2345-2357.

Møller, A.P. 1994. Parasites as an environmental component of reproduction in birds as exemplified by the swallow Hirundo rustica. Ardea 82(1):161-172.

615 Møller, A.P. 2010. Host-parasites interactions and vectors in the barn swallow in relation to climate change. Global Change Biology 16(4):1158-1170.

Møller, A. P, Garamszegi, L. Z. 2010. Immune defense and invasion. En: Morand, S., Krasnov, B. (eds.), The geography of host-parasite interactions, pp. 205-215. Oxford University Press, Oxford, Reino Unido.

Møller, A.P., Merino, S., Soler, J.J., Antonov, A., Badás, E.P., Calero-Torralbo, M.A., et al. 2013. Assessing the effects of climate on host-parasite interactions: A comparative study of European birds and their parasites. PLoS One 8:e82886.

Møller, A.P., Saino, N., Adamík, P., Ambrosini, R., Antonov, A., Campobello, D., et al. 2011. Rapid change in host use of the common cuckoo Cuculus canorus linked to climate change. Proceedings of the Royal Society of London B: Biological Sciences 278(1706):733-8.

Møller, A.P., Soler, J.J. 2012. A coevolutionary framework based on temporal and spatial ecology of host-parasite interactions: a missing link in studies of brood parasitism. Chinese Birds 3(4):259-273.

Morand, S. 2015. (macro-) Evolutionary ecology of parasite diversity: From determinants of parasite species richness to host diversification. International Journal for Parasitology: Parasites and Wildlife 4(1):80-87.

Mouritsen, K.N., Poulin, R. 2002. Parasitism, climate oscillations and the structure of natural communities. Oikos 97(3): 462-468. implications for host life history evolution. Oikos 97(2):223-228. 
Murdock, C.C., Foufopoulos, J., Simon, C.P. 2013. A transmission model for the ecology of an avian blood parasite in a temperate ecosystem. PLoS One 8(9):e76126.

Musgrave, K., Bartlow, A.W., Fair, J.M. 2019. Long-term variation in environmental conditions 640 influences host-parasite fitness. Ecology and evolution 9(13):7688-7703.

Newman, E.A., Eisen, L., Eisen, R.J., Fedorova, N., Hasty, J.M., Vaughn, C., Lane, R.S. 2015. Borrelia burgdorferi sensu lato spirochetes in wild birds in Northwestern California: Associations with ecological factors, bird behavior and tick infestation. PLOS One 10(2):e0118146.

645 Niebuhr, C.N., Poulin, R., Tompkins, D.M. 2016. Is avian malaria playing a role in native bird declines in New Zealand? Testing hypotheses along an elevational gradient. PLoS One 11(11):e0165918.

Oakgrove, K.S., Harrigan, R.J., Loiseau, C., Guers, S., Seppi, B., Sehgal, R.N. 2014. Distribution, diversity and drivers of blood-borne parasite co-infections in Alaskan bird populations. International journal for parasitology 44(10):717-727.

O'Connor, E. A., Cornwallis, C. K., Hasselquist, D., Nilsson, J-Å, Westerdahl, H. 2018. The evolution of immunity in relation to colonization and migration. Nature Ecology and Evolution 2(5):841-849.

Ogden, N.H., Barker, I.K., Francis, C.M., Heagy, A., Lindsay, L.R., Hobson, K.A. 2015. How far north are migrant birds transporting the tick Ixodes scapularis in Canada? Insights from stable hydrogen isotope analyses of feathers. Ticks and Tick-Borne Diseases 6(6):71520.

Ogden, N.H., Bigras-Poulin, M., Hanincová, K., Maarouf, A., O'Callaghan, C.J., Kurtenbach, K. 2008. Projected effects of climate change on tick phenology and fitness of pathogens transmitted by the North American tick Ixodes scapularis. Journal of Theoretical Biology 254(3):621-32.

Ogden, N.H., Maarouf, A., Barker, I.K., Bigras-Poulin, M., Lindsay, L.R., Morshed, M.G., et al. 2006. Climate change and the potential for range expansion of the Lyme disease vector Ixodes scapularis in Canada. International journal for parasitology 36(1):63-70.

665 Pachauri, R.K., Allen, M.R., Barros, V.R., Broome, J., Cramer, W., Christ, R., et al. 2014. Climate change 2014: synthesis report. Contribution of Working Groups I, II and III to the fifth assessment report of the Intergovernmental Panel on Climate Change. IPCC. Geneva, Suiza. http://www.ipcc.ch/report/ar5/syr/

Parmesan, C. 2006. Ecological and evolutionary responses to recent climate change. Annual Review of Ecology, Evolution and Systematics 37:637-669. 
Patz, J.A., Epstein, P.R., Burke, T.A., Balbus J.M. 1996. Global climate change and emerging infectious diseases. Jama 27583:217-223.

Polley, L., Thompson, R.A. 2009. Parasite zoonoses and climate change: molecular tools for tracking shifting boundaries. Trends in Parasitology 25(6):285-291.

Poulin, R., Mouritsen, K.N. 2005. Climate change, parasitism and the structure of intertidal ecosystems. Journal of Helminthology 80(2):183-191.

Price, P.W. 1980. Evolutionary Biology of Parasites. Princeton University Press, Princeton, Estados Unidos de América.

Pulgarín-R, P.C., Gómez, J.P., Robinson, S., Ricklefs, R.E., Cadena, C.D. 2018. Host species, 680 and not environment, predicts variation in blood parasite prevalence, distribution, and diversity along a humidity gradient in northern South America. Ecology and evolution 8(8):3800-3814.

Randolph, S. 2009. Epidemiological consequences of the ecological physiology of ticks. Advances in Insect Physiology 37:297-339.

Reiling, S.J., Dixon, B.R. 2019. Zoonotic diseases: Toxoplasma gondii: How an Amazonian parasite became an Inuit health issue. Canada Communicable Disease Report 45(78):183.

Root, T.L., Price, J.L., Hall, K.R., Schneider, S.H., Rosenzweig, C., Pounds, J.L. 2003. Fingerprints of global warming on wild animals and plants. Nature 421(6918):57-60.

690 Rosenzweig, C., Casassa, G., Karoly, D.J., Imeson, A., Liu, C., Menzel, A., et al. 2007. Assessment of observed changes and responses in natural and managed systems. En: Parry, M.L., Canziani, O.F., Palutikof, J.P., van der Linden, P.J., Hanson, C.E. (eds.), Climate change 2007: impacts, adaptation and vulnerability. Contribution of Working Group II to the Fourth Assessment Report of the Intergovernmental Panel on Climate Change, pp. 79-131. Cambridge University Press, Cambridge, Reino Unido.

Rotics, S., Turjeman, S., Kaatz, M., Resheff, Y., Zurell, D., Sapir, N., et al. 2017. Wintering in Europe instead of Africa enhances juvenile survival in a long-distance migrant. Animal Behaviour 126:79-88.

Roulin, A. 1999. Fécondité de la mouche Carnus hemapterus, parasite des jeunes chouettes effraies (Tyto alba). Alauda 67(3):205-212.

Saino, N., Rubolini, D., Lehikoinen, E., Sokolov, L.V., Bonisoli-Alquati, A., Ambrosini, R., et al. 2009. Climate change effects on migration phenology may mismatch brood parasitic cuckoos and their hosts. Biology Letters 5(4):539-41. 
Santiago-Alarcón, D., Palinauskas, V., Schaefer, H.M. 2012. Diptera vectors of avian 705 Haemosporidian parasites: untangling parasite life cycles and their taxonomy. Biological Reviews 87(4):928-964.

Santos, J.L. 1994. Nutrition, infection, and immunocompetence. Infectious disease clinics of North America 8(1):243-267.

Saunders, L.M., Tompkins, D.M., Hudson, P.J. 2000. The role of oxygen availability in the embryonation of Heterakis gallinarum eggs. International Journal for Parasitology 30(14):1481-1485.

Sehgal, R., Buermann, W., Harrigan, R., Bonneaud, C., Loiseau, C., Chasar, A., et al. 2011. Spatially explicit predictions of blood parasites in a widely distributed African rainforest bird. Proceedings of the Royal Society of London B: Biological Sciences 278(1708):1025e1033.

Shaman, J., Day, J.F. 2007. Reproductive phase locking of mosquito populations in response to rainfall frequency. PLoS one 2(3): e331.

Shaw, A.K., Binning, S.A. 2016. Migratory recovery from infection as a selective pressure for the evolution of migration. The American Naturalist 187(4):491-501.

720 Smith, R.N., Cain, S.L., Anderson, S.H., Dunk, J.R., Williams, S. 1998. Blackfly induced mortality of nestling red-tailed hawks. The Auk 115(2):368-375.

Teplitsky, C., Mills, J.A., Alho, J.S., Yarrall, J.W., Merilä, J. 2008. Bergmann's rule and climate change revisited disentangling environmental and genetic responses in a wild bird population. Proceedings of the National Academy of Sciences 105(36):13492-13496.

725 Tompkins, D.M., Gleeson, D.M. 2006. Relationship between avian malaria distribution and an exotic invasive mosquito in New Zealand. Journal of the Royal Society of New Zealand 36(2):51-62.

Tong, S.L., Hu, W. 2001. Climate variation and incidence of Ross River virus in Cairns, Australia: A time-series analysis. Environmental Health Perspectives 109(12):1271-1273.

730 Travers, M.A., Le Goïc, N., Huchette, S., Koken, M., Paillard, C. 2008. Summer immune depression associated with increased susceptibility of the European abalone, Haliotis tuberculata to Vibrio harveyi infection. Fish \& shellfish immunology 25(6):800-808.

Van Riper III, C., van Riper, S.G., Goff, M.L., Laird, M. 1986. The epizootiology and ecological significance of malaria in Hawaiian land birds. Ecological monographs 56(4):327-344.

van Rooyen, J., Lalubin, F., Glaizot, O., Christe, P. 2013. Altitudinal variation in haemosporidian parasite distribution in great tit populations. Parasites \& Vectors 6(1):139. 
Walsh, K.J.E., Ryan, B.F. 2000. Tropical Cyclone Intensity Increase near Australia as a Result of Climate Change. Journal of Climate 13(16):3029-3036.

Walther, G.R., Post, E., Convey, P., Menzel, A., Parmesan, C., Beebee, T.J. et al. 2002. Ecological responses to recent climate change. Nature 416(6879):389-395.

Warner, R.E. 1968. The role of introduced diseases in the extinction of the endemic Hawaiian avifauna. The Condor 70(2):101-120.

Wegbreit, J., Reisen, W.K. 2000. Relationships among weather, mosquito abundance and encephalitis virus activity in California: Kern County 1990-98. Journal of the American Mosquito Control Association 16(1):22-27.

Wegner, K.M., Kalbe, M., Milinski, M., Reusch, T.B. 2008. Mortality selection during the 2003 European heat wave in three-spined sticklebacks: effects of parasites and MHC genotype. BMC Evolutionary Biology 8(1):124.

Wiedenfeld, D.A., Fessl, B., Kleindorfer, S., Valarezo, J.C. 2007. Distribution of the introduced 750 parasitic fly Philornis downsi (Diptera, Muscidae) in the Galápagos Islands. Pacific conservation biology 13(1):14-19.

Wu, X., Lu, Y., Zhou, S., Chen, L., Xu, B. 2016. Impact of climate change on human infectious diseases: Empirical evidence and human adaptation. Environment international 86:14-23.

Zamora-Vilchis, I., Esparza-Salas, R., Johnson, C.N., Williams, S.E., Endler, J.A. 2013. Parasite-mediated diversity and selection of MHC genes of birds distributed along an altitudinal gradient: implications for disease impact in a warming climate. En: ZamoraVilchis, I. (eds.), Host-parasite interactions: bird immune genes, blood parasites and climate change implications. Tesis de Doctorado, Universidad James Cook, Queensland, Australia.

Zamora-Vilchis, I., Williams, S.E., Johnson, C.N. 2012. Environmental temperature affects prevalence of blood parasites of birds on an elevation gradient: implications for disease in a warming climate. PloS one 7(6).

Zhou, G., Minakawa, N., Githeko, A.K., Yan, G.Y. 2004. Association between climate variability and malaria epidemics in the East African highlands. Proceedings of the National Academy of Sciences 101(8):2375-2380. 
Tabla 1. Relación entre las variables climáticas precipitación/humedad y temperatura y la abundancia de diferentes vectores y parásitos. El color verde representa una relación positiva entre las variables, el color amarillo representa una relación positiva o negativa en función del hábitat geográfico y el color rojo representa una relación negativa.

Table 1. Relationship among climatic variables precipitation/humidity and temperature with the abundance of different vectors and parasites. The green colour represents a positive relationship among variables, the yellow colour represents a positive or negative relationship depending on the geographical habitat and the red colour represents a negative relationship.

\begin{tabular}{|c|c|c|c|}
\hline $\begin{array}{l}\text { Variables } \\
\text { climáticas }\end{array}$ & $\begin{array}{c}\text { Abundancia } \\
\text { Vectores }\end{array}$ & Abundancia Parásitos & Referencias \\
\hline $\begin{array}{l}\text { Precipitaciones } \\
\text { / Humedad }\end{array}$ & $\begin{array}{c}\text { Insectos } \\
\text { nematóceros }^{1}\end{array}$ & $\begin{array}{l}\text { Hemoparásitos }^{2} \\
\text { Ácaros }^{3} \\
\text { Mosca Philornis } \\
\text { torquans }^{4}\end{array}$ & 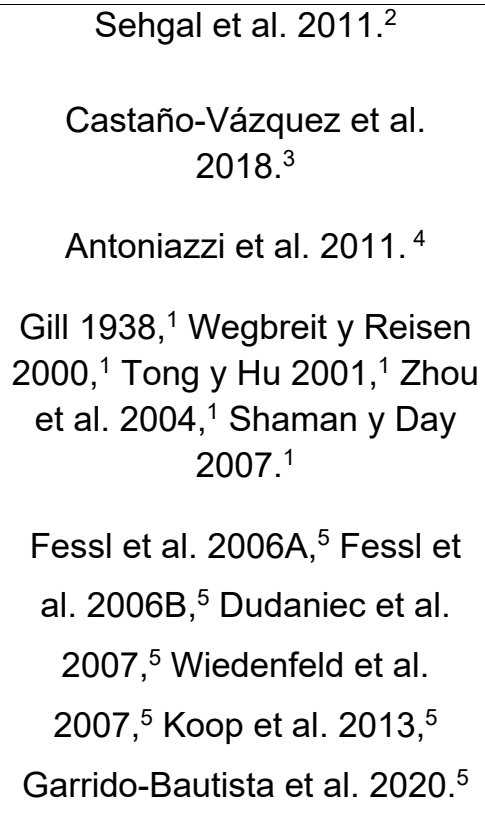 \\
\hline Temperatura & $\begin{array}{c}\text { Insectos } \\
\text { nematóceros }\end{array}$ & $\begin{array}{c}\text { Nematodo H. gallinarum } \\
\text { Hemoparásitos }^{8} \\
\text { Mosca Trypocalliphora } \\
\text { braueri }^{9} \\
\text { Ácaros }{ }^{11} \\
\text { Moscas (Protocalliphora, } \\
\text { Philornis downsi) }\end{array}$ & $\begin{array}{c}\text { Saunders et al. 2000. } \\
\text { Garamszegi 2011, }{ }^{6} \text { Sehgal et } \\
\text { al. 2011, Zamora-Vilchis et al. } \\
\text { 2012, }{ }^{8} \text { González-Quevedo et } \\
\text { al. 2014, }{ }^{8} \text { Niebuhr et al. } \\
\text { 2016. }{ }^{8} \\
\text { Musgrave et al. 2019. }{ }^{9} \\
\text { Bennett y Whitworth } 1991,{ }^{12} \\
\text { Dawson et al. 2005, }{ }^{12} \\
\text { Dudaniec et al. 2007, }{ }^{12} \\
\text { Meléndez et al. 2014, }{ }^{11} \\
\text { Castaño-Vázquez et al. }\end{array}$ \\
\hline
\end{tabular}




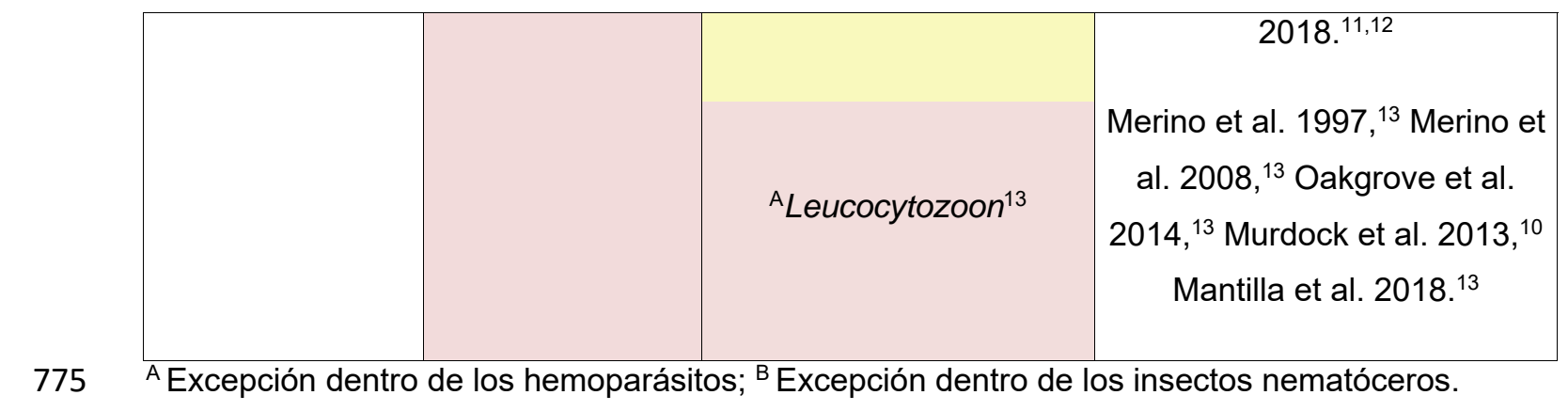


Tabla 2. Efectos del cambio climático sobre la fenología de los parásitos y hospedadores. El color verde representa un avance en la fenología de los organismos y el color amarillo representa resultados discrepantes según la especie y la región geográfica.

Table 2. Effects of climate change on parasite and host phenology. The green colour represents an advance in the phenology of these organisms and the yellow colour represents discrepant results depending on the species and the geographical region.

\begin{tabular}{|c|c|c|c|}
\hline $\begin{array}{c}\text { Cambios en el } \\
\text { clima }\end{array}$ & $\begin{array}{c}\text { Fenología de los } \\
\text { parásitos }\end{array}$ & $\begin{array}{c}\text { Fenología de } \\
\text { los } \\
\text { hospedadores }\end{array}$ & Referencias \\
\hline $\begin{array}{c}\text { Temperaturas } \\
\text { mayores y } \\
\text { primaveras } \\
\text { tempranas }\end{array}$ & $\begin{array}{c}\text { Mosca Ornithomyia } \\
\text { avicularia }^{1} \\
\text { Mosca Carnus } \\
\text { hemapterus }^{2} \\
\text { Garrapata Ixodes } \\
\text { scapularis }^{3} \\
\text { Cuco común Cuculus } \\
\text { canorus }^{4}\end{array}$ & $\begin{array}{l}\text { Hospedadores } \\
\text { de Cuculus } \\
\text { canorus y } \\
\text { Clamator } \\
\text { glandarius }^{5}\end{array}$ & $\begin{array}{c}\text { Møller 2010. }{ }^{1} \\
\text { Calero-Torralbo } 2011,{ }^{2} \\
\text { Amat-Valero et al. } 2013 .^{2} \\
\text { Ogden et al. } 2008,{ }^{3} \text { Levi } \\
\text { et al. } 2015 .{ }^{3} \\
\text { Saino et al. 2009, }{ }^{5} \text { Møller } \\
\text { y Soler 2012, }{ }^{4} \text { Avilés et al. } \\
\text { 2014, }{ }^{5} \text { Barrett } 2014,{ }^{5} \\
\text { Berkowic et al. } 2015,{ }^{4,5} \\
\text { Dyrcz y Czyz 2018. }{ }^{5}\end{array}$ \\
\hline
\end{tabular}


Tabla 3. Efectos del cambio climático sobre los rangos de distribución de parásitos, vectores y hospedadores. El color verde representa un aumento en el rango de distribución y el color rojo representa una disminución del rango de distribución.

Table 3. Effects of climate change on parasite, vector and host distribution ranges. The green colour represents an increase in the distribution range and the red colour represents a decrease in the distribution range.

\begin{tabular}{|c|c|c|c|c|}
\hline \multirow[b]{2}{*}{$\begin{array}{c}\text { Cambios en } \\
\text { el clima }\end{array}$} & \multicolumn{3}{|c|}{ Rango de distribución } & \multirow[b]{2}{*}{ Referencias } \\
\hline & Vectores & Parásitos & Hospedadores & \\
\hline $\begin{array}{c}\text { Aumento de } \\
\text { la } \\
\text { temperatura }\end{array}$ & $\begin{array}{c}\text { Insectos } \\
\text { nematóceros }{ }^{1}\end{array}$ & $\begin{array}{l}\text { Garrapatas }^{2} \\
\text { Toxoplasma } \\
\text { gondii }^{3} \\
\text { Hemoparásitos } \\
\text { 4 }\end{array}$ & $\begin{array}{c}\text { Aves } \\
\text { migratorias }^{6} \\
\text { Felinos } \\
\text { silvestres }^{7}\end{array}$ & $\begin{array}{c}\text { Hasle et al. 2011, }{ }^{2,6} \\
\text { Leighton et al. 2012, }{ }^{2,6} \\
\text { Jenkins et al. 2013, }{ }^{3,7} \\
\text { Ogden et al. } 2015,{ }^{2} \text { Reiling } \\
\text { y Dixon } 2019 .^{6} \\
\text { Tompkins y Gleeson } \\
\text { 2006, }{ }^{1,4} \text { Atkinson } 2008,{ }^{1,4} \\
\text { Lovejoy } 2008,{ }^{1,4} \text { Loiseau et } \\
\text { al. } 2012,{ }^{4} \text { Zamora-Vilchis } \\
\text { et al. } 2012,{ }^{1,4} \text { Loiseau et } \\
\text { al. } 2013,{ }^{4} \text { Atkinson et al. } \\
\text { 2014, }{ }^{1} \text { Liao et al. } 2015,{ }^{1} \\
\text { Chapa-Vargas et al. } 2020 .{ }^{4}\end{array}$ \\
\hline
\end{tabular}

Excepción dentro de los hemoparásitos. 
Tabla 4. Efecto de las variaciones en el clima y su repercusión sobre la virulencia de los parásitos y la inmunocompetencia de las aves. El color verde y el color rojo representan un efecto positivo y negativo, respectivamente, sobre las dos variables (virulencia de los parásitos e inmunocompetencia de las aves).

Table 4. Effect of variations in climate and its repercussion on virulence of parasites and immunocompetence of birds. The green colour and the red colour represent a positive and negative effect, respectively, on the two variables (virulence of parasites and immunocompetence of the birds).

\begin{tabular}{|c|c|c|c|}
\hline $\begin{array}{l}\text { Cambios en el } \\
\text { clima }\end{array}$ & $\begin{array}{l}\text { Virulencia de los } \\
\text { parásitos }\end{array}$ & $\begin{array}{c}\text { Inmunocompetencia } \\
\text { de las aves }\end{array}$ & Referencias \\
\hline $\begin{array}{c}\text { Aumento de la } \\
\text { temperatura } \\
y \\
\text { cambios en la } \\
\text { estacionalidad } \\
\text { de las } \\
\text { precipitaciones }\end{array}$ & $\begin{array}{c}\text { Ciclo de vida de los } \\
\text { parásitos más rápido. }{ }^{2} \\
\text { Hospedadores } \\
\text { inmunodeprimidos. }^{3} \\
\text { Expansión de parásitos } \\
\text { generalistas. }{ }^{4} \\
\text { Menor actividad de } \\
\text { búsqueda de } \\
\text { hospedadores en } \\
\text { garrapatas. }{ }^{5}\end{array}$ & $\begin{array}{c}\text { Efecto de nuevos } \\
\text { parásitos por el } \\
\text { aumento del rango de } \\
\text { distribución. }^{6} \\
\text { Mayor densidad de } \\
\text { parásitos. }^{7} \\
\text { Menor disponibilidad de } \\
\text { alimento. }^{8} \\
\text { Estrés térmico. }^{9}\end{array}$ & 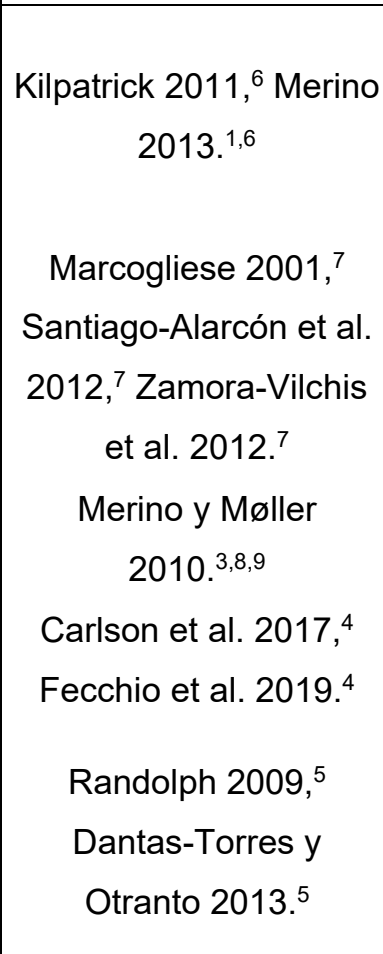 \\
\hline
\end{tabular}

https://www.nature.com/scitable/knowledge/evolution-13228138/ 